\title{
Discussion on Production Technology of Formaldehyde Purification and Anti-cracking Ecological Board and the Intuitive Display Experiment
}

\author{
Liu Yaunqiang ${ }^{1}$, Liu Yixin ${ }^{2,}$, , Weng Yaolie ${ }^{1}$, Guo Bingtuo ${ }^{1}$, Ye Jiaoyou ${ }^{1}$ \\ ${ }^{1}$ Dehua Tubaobao New Decorative Material Limited Company, Deqing, China \\ ${ }^{2}$ School of Laboratory Medicine, Tianjin Medical University, Tianjin, China
}

Email address:

fxzlyq@126.com (Liu Yaunqiang),821672909@qq.com (Liu Yixin)

${ }^{*}$ Corresponding author

\section{To cite this article:}

Liu Yaunqiang, Liu Yixin, Weng Yaolie, Guo Bingtuo, Ye Jiaoyou. Discussion on Production Technology of Formaldehyde Purification and Anti-cracking Ecological Board and the Intuitive Display Experiment. American Journal of Agriculture and Forestry.

Vol. 6, No. 4, 2018, pp. 98-102. doi: 10.11648/j.ajaf.20180604.16

Received: July 2, 2018; Accepted: July 20, 2018; Published: August 23, 2018

\begin{abstract}
The formaldehyde purification and anti-cracking ecological board has the function of anti-cracking and removing formaldehyde, which is improved on the basis of the production process of ordinary ecological boards. Its key processes are different from the ordinary ecological board including the secondary adhesive mixing, secondary adhesive dipping, resin-impregnated paper storage, optimization of balanced laminate (1.0mm hight density fibreboard, HDF), stickers, hot pressing and so on. In addition, the core technology parameters of formaldehyde purifying ecological board involves several aspects such as the glue preparation ratio, curing time, dipping amount, storage environment of the resin-impregnated paper, hot-pressing process of the balanced laminate layer and the resin-impregnated paper. The anti-cracking property of the formaldehyde purification and anti-cracking ecological board under this process is greater than grade 4 . The main active ingredient of formaldehyde purification and anti-cracking eco-board are chitin and nano-silicon, etc. The negative electric field generated by nano-crystalline silicon wafers adsorbs gas molecules such as formaldehyde, the active amino groups in chitin decompose these substances into water molecules, and the nano-silicon wafers also function as bactericidal agents to achieve the effect of air purification. After testing, the purification efficiency of formaldehyde purifying and anti-cracking ecological board $\geq$ $75 \%$, and the formaldehyde purifying effect lasting $\geq 60 \%$. In the intuitive formaldehyde purification experiment, the formaldehyde level can be approximately decreased to $50 \%$ within $10 \mathrm{~min}$ and reduced to $0.3 \mathrm{mg} / \mathrm{m}^{3}$ after $1 \mathrm{~h}$ in the newly prepared formaldehyde purification and anti-cracking ecological board samples.
\end{abstract}

Keywords: Formaldehyde Purification, Anti-cracking, Ecological Board, Production Technology,

Optimization of Balanced Laminate, Formaldehyde Purification Performance, Intuitive Display Experiment

\section{Introduction}

With blockboard or ordinary plywood as the substrate, the surface-laminated melamine-impregnated veneer paper is commonly known as ecological panels. It is currently one of the most used boards in the furniture and building materials industry. After several years of development, there are now thousands of resin-impregnated films printed with water-based ink and impregnated with melamine resin, which are environmentally friendly and paintless. There are various types of textures, and the textures and colors include almost all kinds of land-based wood species, accompanied with the diversification of soft light, colors, and relief, giving interior decoration designers a broader space for imagination. Regardless of the whole house model customization, or individual needs, the ecological board can better meet the needs of people's indoor applications.

The quality of the air environment is a focus issue of the entire society. The national environmental protection storm is intended to restore the blue sky and focus on the outdoor PM2.5 problem. However, indoor formaldehyde, TVOC, and excessive bacterial concentrations seriously affect people's 
physical and mental health. Ordinary wood-based panel furniture panels use amino resin thermosetting to achieve the bonding effect. Although the bonding performance is excellent, the free formaldehyde will deteriorate the indoor air quality [1], which will breed health hazards for young and old, infirm or pregnant women. In addition, fabric sofas, laminate flooring, wallpapers, textile wall coverings, mattresses, etc. used in interiors will also release formaldehyde. Therefore, the functional plate that can absorb and decompose the formaldehyde in the air is the demand for people's health, and it is also the direction for the development of furniture plates.

Conventional ecological board structure [2-5] is: resin-impregnated paper + balanced laminate + base material + balanced laminate + resin-impregnated paper. The balanced laminate close to the impregnated paper is $0.4 \sim 1.0 \mathrm{~mm}$ thick poplar veneer or technical wood veneer, and its anisotropy is obvious. Its easy for it to deform and produce great internal stressin the case of humidity or temperature changes. Therefore, there is the hidden danger of surface crack. On the premise of keeping the base material layer of the ecological board unchanged, this program optimizes the balance laminate and changes the $0.4 \sim 1.0 \mathrm{~mm}$ veneer of the original structure into $1.0 \mathrm{~mm}$ HDF (density is greater than $0.90 \mathrm{~g} / \mathrm{cm}^{3}$ ), which increases the surface density of the substrate and makes it uniform in texture. This program thoroughly solves the cracking problem caused by internal stress due to hot pressing of the balance layer.

This article discusses the production process and purification principles of the formaldehyde purification and anti-cracking eco-board, and introduces the intuitive display method of its performance to enhance consumers and their peers' understanding of the product.

\section{Production Technology of Formaldehyde Purification and Anti-cracking Ecological Board}

\subsection{Production Process}

Formaldehyde purification and anti-cracking eco-board production process includes: 1) Preparation of formaldehyde purification impregnated film paper; 2) Pressing the formaldehyde purification impregnated film paper on the composite material of $1.0 \mathrm{~mm}$ HDF with plywood or blockboard cores, giving the ecological board formaldehyde purification and anti-cracking performance.

1) The production process of formaldehyde purifying impregnated film paper is as Figure 1

adhesive mixing 1

adhesive mixing 2

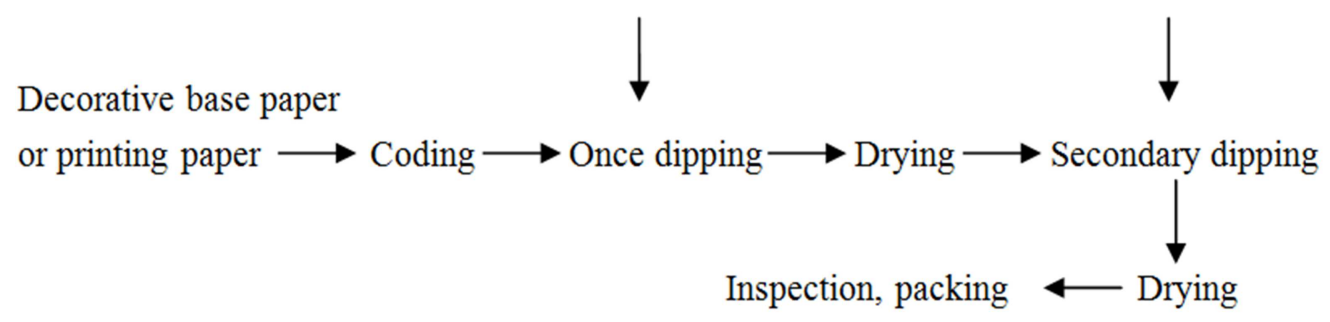

Figure 1. Process chart of formaldehyde purification impregnating paper.

2) The production process of formaldehyde purification ecological board is as Figure 2

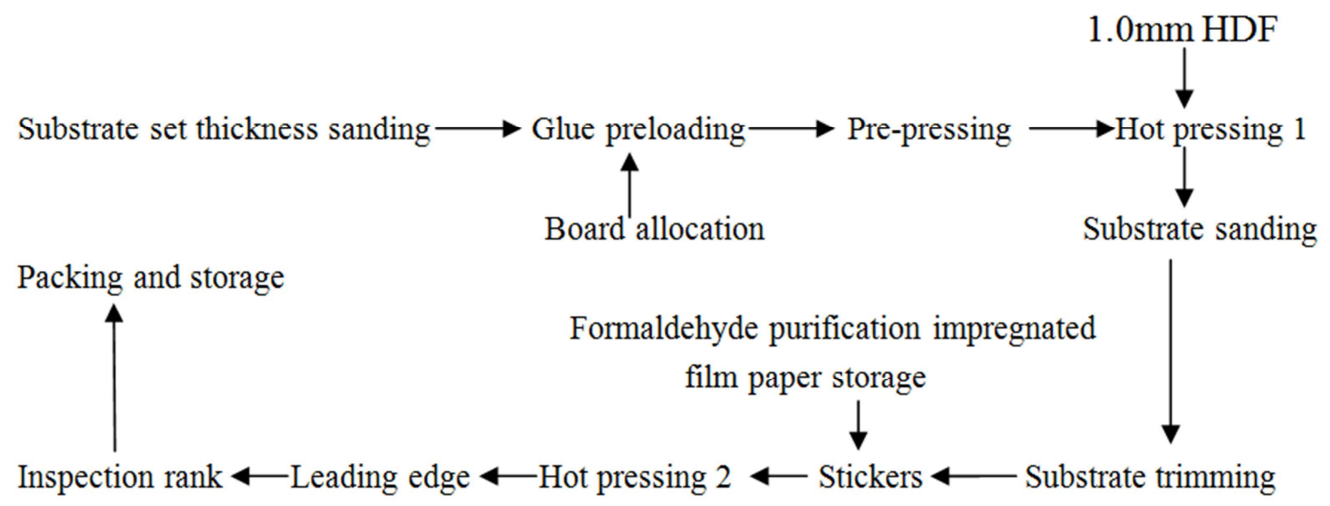

Figure 2. Production process chart of formaldehyde purifying ecologic board.

\subsection{Key Process Plan}

The production of formaldehyde purifying and anti-cracking eco-board is based on the improvement of the production process of ordinary impregnated film paper and its eco-board. The formaldehyde scavenging additive is applied in adhesive mixing 2 and secondary adhesive dipping processes. The main components of formaldehyde scavenging are chitin and nanocrystalline silicon and other natural 
substances [6-8]. The active ingredient is attached to the impregnated paper along with the melamine formaldehyde resin solution. The surface layer is dried and pre-cured to form a formaldehyde purifying impregnated film. The key process of the entire formaldehyde purification and anti-cracking ecological board is: adhesive mixing 2 , secondary adhesive dipping, the storage of formaldehyde purification impregnated plastic paper, hot-pressing 1, sticking papers, hot-pressing 2 . The specific process plan is as follows:

1) Adhesive mixing 2: After quantifying the melamine formaldehyde resin solution, inject the melamine formaldehyde resin solution into the adjusting glue tank, and adding the auxiliaries such as release agent, penetrant, dedusting agent and the like, and the formaldehyde purifying additive and curing agent according to the relative proportions. After stirring, we test the curing time of the glue. The curing time is approximately 7 minutes in the summer and 5.5 minutes in the winter. Continue stirring to ensure that all additives are dissolved and set aside.

2) Secondary adhesive dipping: The prepared glue is connected with the rubber pump, glue tank and glue reflow system respectively. Parameters such as the amount of dipping and precure percent are adjusted according to the characteristics of the base paper and the melamine formaldehyde resin solution. The amount of secondary adhesive dipping is about $65 \mathrm{~g} / \mathrm{m}^{2}$.

3) Impregnated film paper storage: set up impregnated film paper storage warehouse and equipped with air conditioning facilities. The storage environment temperature is lower than $25^{\circ} \mathrm{C}$ and the humidity is less than $75 \%$, Storage time does not exceed 60 days. It is forbidden to place the impregnated paper next to the hot press in hot summer.

4) Hot pressing 1: The surface of plywood or blockboard is sanded to a certain thickness, then both sides of substrate are coated with adhesive and compounded with $1.0 \mathrm{~mm}$ HDF, which are followed by cold pressure and hot pressure successively. The hot-pressing process is: unit pressure $0.8 \sim$ 1.0MPa, temperature $125 \pm 5^{\circ} \mathrm{C}$, time $3 \sim 5 \mathrm{~min}$.

5) Stickers: Apply melamine impregnated film on both sides of the substrate as required. The impregnated paper must completely cover the substrate.

6) Hot pressing 2: Using a single-sided hot-pressing process, that is, one side of hot pressing to complete cooling and then pressing the other side. The hot-pressing process is: unit pressure $0.6 \sim 1.0 \mathrm{MPa}$, temperature $125 \pm 5{ }^{\circ} \mathrm{C}$, time $8 \sim$ $10 \mathrm{~min}$. After hot pressing, the plate surface should be smooth and clean, with no frosting mark and water mark, and bright spots, and no waste indentation and other defects.

\subsection{Performance Testing}

The surface performance of formaldehyde purifying and anti-cracking eco-board is tested based on national standard GB/T 34722-2017 including the surface adhesive strength, surface resistance to cold and heat cycles, surface wear resistance, surface scratch resistance, surface crack resistance, surface pollution corrosion resistance, surface resistance to dry heat, light resistance color fastness and other indicators.
The formaldehyde purification performance is tested based on JC/T1074-2008 'indoor air purification function coating material purification performance' including formaldehyde purification efficiency and formaldehyde purification effect of durability. Alternatively all the indicators of formaldehyde purification and anti-cracking eco-board are tested according to $\mathrm{Q} / \mathrm{TBB}$ 0022-2016 'impregnated film coated plywood'. After testing, the three purification indicators of formaldehyde purification and anti-cracking of ecological panels are: formaldehyde purification efficiency $\geq 75 \%$, formaldehyde purification effect persistence $\geq 60 \%$, the anti-cracking performance up to level 5 .

\subsection{Formaldehyde Purification Mechanism}

The main active ingredient of formaldehyde purification and anti-cracking eco-board are chitin and nano-silicon, etc. Chitin is an organic natural material with a high molecular weight. It has been recognized by the scientific community as the sixth largest element of human life. It has a stable unsaturated positive charge structure and a strong adsorption capacity for small molecular free substances. Nano wafers have about 20,000 negative charges/unit. The strong negative electric field can adsorb formaldehyde and other gas molecules in the air to the surface of the plate. The active amino in the chitin then decomposes these substances into water molecules, and the nano-silicon tablets also function as a sterilizer, thereby achieving the effect of air purification.

The process of decomposition of formaldehyde by chitin is as Figure 3.

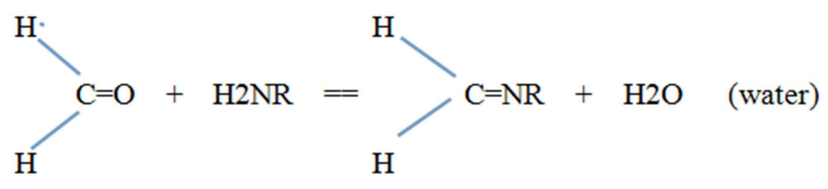

Figure 3. Reaction formula for the reaction of chitin to formaldehyde.

Chitin is an extract of shrimp and crab shells. It is environmentally friendly and possesses high medical value. It is the only animal cellulose found in nature so far and one of the basic elements of life. Deacetyl chitin is chitosan and has physiological activity. It is extensively applied in medicine and food.

At present, under the pressure of the national mandatory standard GB50325, real estate developers have already felt the emergency of air quality requirements. When air quality is substandard, it cannot pass the acceptance. Even if it is passed by chance, the consumer can hire an air quality supervision department to perform the inspection. The consumer may demands a claim or rework and even check out. In 2017, the delivery mode of hardcover houses will be launched nationwide. Whether soft or hard decoration, strict implementation of the national mandatory standard GB50325 is required. The concentration of formaldehyde in the air shall be $\leq 0.08 \mathrm{mg} / \mathrm{m}^{3}$ and TVOC shall be less than $0.5 \mathrm{mg} / \mathrm{m}^{3}$ [9]. Non-environmentally friendly products will have no place and air purification products are the first choice for people to decorate. 


\section{Formaldehyde Purification Performance Display Test}

\subsection{Test Materials and Instruments}

1) Test materials

Formaldehyde purification and anti-cracking eco-board sample: Dehua TBB Decoration New Material Co., Ltd., specifications $100 * 150 \mathrm{~mm}$, quantity 4 pieces.

Formaldehyde solution: Dehua TBB Adhesive Material Co. Ltd., concentration 37\%. Medical absorbent cotton swab: Size $15 \mathrm{~cm}$

2) Main equipment

Formaldehyde detection test box: made of tempered glass, thickness $4 \mathrm{~mm}$, size $300 * 300 * 300 \mathrm{~mm}$.

Computer chassis fan: Model 5V-DC, ROHS.

Formaldehyde detector: built-in electrochemical sensor and high-precision operational amplifier chip, model BGFM-06, produced by Beigu Technology Co., Ltd.

Medical pipettes: specifications $1 \mathrm{ml}$, with needle specifications $0.4 \mathrm{~mm}$, model $0.4 * 15 \mathrm{RWLB}$.

\subsection{Test Methods}

\subsubsection{Test Principle}

In a sealed, tempered glass box, an open formaldehyde tester was placed showing a formaldehyde concentration of less than $0.1 \mathrm{mg} / \mathrm{m}^{3}$. A small hole is opened on the side, and the medical absorbent cotton injected with $0.02 \sim 0.03 \mathrm{ml}$ formaldehyde solution is inserted into the box, and the fan is stirred to open the mixed air in the box. When the formaldehyde concentration rises to $1.0 \mathrm{mg} / \mathrm{m}^{3}$, the cotton swab is removed and the formaldehyde purification and anti-cracking ecological board was quickly put into it. Then sealing the test box. Finally, the decreasing rate of formaldehyde concentration in the box and the effect of aldehyde removal can be observed.

Active ingredients on the surface of the formaldehyde purification and anti-cracking ecological board such as chitin and nano-silicon, can chemically react with the air containing high formaldehyde concentration quickly. Formaldehyde is decomposed into water during this process, and the water evaporates into the mixed air, making the formaldehyde purification effect visible.

\subsubsection{Operation Process}

1) Prepare the formaldehyde purification and anti-cracking ecological board sample and close the sides with formaldehyde -free aluminum tape. Wipe the surface and keep it clean.

2) Open the formaldehyde tester and observe that the indoor formaldehyde concentration is within the qualified value 0.1 $\mathrm{mg} / \mathrm{m}^{3}$. Put it into the formaldehyde test box, and maintain no significant change in the formaldehyde concentration.

3) Use a medical pipette to draw $0.5 \mathrm{ml}$ of formaldehyde solution. Inject 0.02 to $0.03 \mathrm{ml}$ onto the prepared cotton swab, and insert the cotton swab into the formaldehyde test box. Open the fan to stir the mixed air in the box, and the concentration of formaldehyde rose to $1.0 \mathrm{mg} / \mathrm{m}^{3}$ in about 2 minutes (at room temperature about $25^{\circ} \mathrm{C}$ ). Medical cotton swabs and formaldehyde test box lids are connected to the box body with a rubber seal.

4) Quickly open the lid, place the sample in the formaldehyde purification eco-plate, and seal the test box. Due to the hysteresis of the electrochemical sensor of the formaldehyde tester, the formaldehyde concentration will still rise after the sample is placed. The highest break point was about $1.2 \mathrm{mg} / \mathrm{m}^{3}$, and then began to decline.

5) Observe the decreasing rate of formaldehyde concentration in the box and the effect of formaldehyde purification.

\subsection{Results and Analysis}

1) Through the above-mentioned visual display test, the freshly prepared formaldehyde purifying and anti-cracking eco-plate sample (the surface has not been contaminated by high-concentration formaldehyde) can remove about $50 \%$ of formaldehyde within 10 minutes, and can be reduced to about $0.3 \mathrm{mg} / \mathrm{m}^{3}$ after 1 hour.

2) In this test, four specimens of a size of $100 * 150 \mathrm{~mm}$ were used, with a total surface area of $1200 \mathrm{~cm}^{2}$, a volume of about $27000 \mathrm{~cm}^{3}$, and a surface-to-volume ratio of $1: 22.5$. When the volume of the box is fixed, the surface area of the sample is increased, and the formaldehyde purification effect is better.

3) The formaldehyde purification and anti-cracking eco-board sample of this test is surrounded by formaldehyde-free aluminum tape to reduce the porosity of the wood part at the edge. When the formaldehyde concentration is high, formaldehyde is adsorbed, and formaldehyde is released at a low concentration after formaldehyde purification. Affects the formaldehyde purification effect of the entire process.

4) This test is a simple operation experiment. It is only to observe whether the product has the function of removing aldehyde. The sensor of the formaldehyde detector used will be interfered by certain external substances. Alcohols, perfumes and other alcohol-containing substances in the air as well as high concentrations of benzene substances, $\mathrm{CO}, \mathrm{H}_{2}$, $\mathrm{SO}_{2}$, smoke etc. all affect the measurement results. Accurate quality testing methods should be based on the national industry standard JC/T1074-2008 "indoor air purification functional coating material purification performance" test two performance indicators, respectively, formaldehyde purification performance and formaldehyde purification effect of durability, detection time is $24 \mathrm{~h}$ and $6 \mathrm{~d}$.

\section{Conclusion}

With the promotion of environmental protection and health of the whole people, formaldehyde purification products have been recognized by people. After testing, the purification efficiency of formaldehyde purifying and anti-cracking ecological board $\geq 75 \%$, and the formaldehyde purifying effect lasting $\geq 60 \%$, meeting the requirements of the standard. Chitin and nano-silicon wafers are used as effective 
components of purifying formaldehyde. The negative electric field generated by nano-crystalline silicon wafers adsorbs gas molecules such as formaldehyde, the active amino groups in chitin decompose these substances into water molecules, and the nano-silicon wafers also function as bactericidal agents to achieve the effect of air purification.

The formaldehyde purifying and anti-cracking eco-board is improved on the basis of ordinary impregnated film paper and its ecological board. The key processes that are different from ordinary eco-boards are: secondary adhesive mixing, secondary adhesive dipping, impregnated film storage, optimization of balanced laminate (1.0mm HDF), sticking papers, hot pressing, etc. The glue modulation ratio, curing time, dipped amount, impregnated paper storage environment and its core processes are hot-pressing process of the balanced laminate and the resin-impregnated paper.

The poplar veneer or technical wood veneer of substrate is relaced by $1.0 \mathrm{~mm}$ HDF. This kind of substrate has uniform thickness and smooth surface and no wave pattern on the surface then is coated with impregnated paper after coating with impregnated paper, which is particularly suitable for custom furniture. Because $1.0 \mathrm{~mm} \mathrm{HDF}$ is homogeneous material and has no structural internal stress, the defects of product cracking are solved. After testing, the anti-cracking performance up to level 5.

Formaldehyde purification visual display test allows people to witness the product removing formaldehyde from air in a short time. The freshly prepared formaldehyde purifying and anti-cracking eco-plate sample (the surface has not been contaminated with high concentrations of formaldehyde) can remove about $50 \%$ of formaldehyde within 10 minutes and the formaldehyde concentration can be reduced to about 0.3 $\mathrm{mg} / \mathrm{m}^{3}$ after 1 hour. There is an error in the displayed data due to the limitations of the electrochemical sensor in the tester and the influence of other mixed gases in the air. Therefore, the results of the visual display test only judge the trend of formaldehyde purification, and more accurate performance determination should be based on the detection of formaldehyde purification efficiency and durability of purification effect following JC/T1074-2008.

\section{Acknowledgements}

This work was supported by 'Development and industrialization of key technologies for functional intelligent household products' of the key research and development projects in zhejiang province. (2017C01117).

\section{References}

[1] WU Zhenhua, LIU Yuanqiang, ZHANG Xiaowei, etc. Formaldehyde Emissions from Impregnated Papers and Blockboards Overlaid with the Papers [J]. China Wood Industry, 2017(6): 39-42.

[2] Wang Xiaoye; Peng Limin; Pang Xiaoren, etc. Situation and Development Trend of Surface Decorated Plywood and Blockboard with Paper Impregnated Thermosetting Resins [J]. China Wood-Based Panels, 2017(2): 9-11.

[3] Fu Jilei, Guo Minghui. Effect of ratio of urea formaldehyde resin in impregnated papers on formaldehyde emission of surface decorated wood-based panels [J]. Journal of Nanjing Forestry University (Natural Sciences), 2014(5): 161-164.

[4] Fu Jilei, Wang Guangsen, Guo Minghui, etc. Prospect of Impregnated Decorative Paper and Surface Decorated Wood-based Panels Industry in China [J]. World Forestry Research, 2014(3): 62-66.

[5] Zhan Xian-xu, Xie Xu-qin, Ye Jiao-you, etc. Technology of Surface Decorated Plywood and Blockboard Overlaid with Impregnated Paper [J]. China Wood Industry, 2016(2): 57-59.

[6] Liu Yuanqiang, Zhan Xianxu, Ye Jiaoyou, etc. A formaldehyde purification ecological board and its preparation method. National invention patent, authorized announcement date: 2017-03-29.

[7] Wan Caichao, Liu Yu, Jiao Yue etc. Effects of Hot-pressing Variables on Formaldehyde Emissions of Melamine Resin Surfaced Particleboard [J]. Forest Engineering, 2014(2): 71-74 +134 .

[8] Chi Yongliang. Study on the Correlation between Desiccator Method and the Perforation Extraction Method for the Determination of Formaldehyde Emission in Medium Density Fiberboard [J]. Forestry Machinery \& Woodworking Equipment, 2015 (3):19-21 + 26.

[9] Wang Xiyuan, Liu Hongkui et al. Specification for Indoor Environmental Pollution Control of Civil Construction Projects. National Standard of the People's Republic of China GB50325-2010, Implementation Date: 2011-06-01. 